\title{
Synthesis of Temperature/pH-Sensitive Hydrogels Containing Disulfide Linkages as Cross-Links and Their Characterization
}

\author{
Hsin-Cheng $\mathrm{CHIU}^{\dagger}$ and Chau-Hui WANG \\ Department of Chemical Engineering, National Chung Hsing University, Taichung 402, Taiwan
}

(Received November 29, 1999)

\begin{abstract}
Temperature/pH-sensitive hydrogels were prepared from the copolymerization of $N$-isopropylacrylamide, $N$-t -butylacrylamide, acrylic acid and methacryloylglycylglycine $p$-nitrophenylester, followed by cross-linking of polymeric precursors with cystamine. The structures of copolymers were characterized by ${ }^{1} \mathrm{H}$ NMR, FT-IR, and GPC. Hydrogels underwent volume change in response to $\mathrm{pH}$ and temperature variation in proportion to content of acrylic acid and $N$-isopropylacrylamide of gels, respectively. Characterization of hydrogels by mechanical measurements under $\mathrm{pH} 7.4$ conditions appears that, owing to the decrease in the modulus of elasticity by extensive swelling of gels, the effective network density is reduced with increasing acrylic acid content at comparable degrees of chemical cross-linking and polymer concentrations. Hydrogels were degraded by 2-mercaptoethanol, L-cysteine and dithioerythritol whereas rapid deswelling behavior occurred upon the exposure of gels to glutathione. The release rate of bovine serum albumin from hydrogels was primarily determined by the swelling extent of hydrogels.
\end{abstract}

KEY WORDS Hydrogels / Temperature / pH-Dependent Swelling / Degradability / Drug Delivery System /

Hydrogels have been receiving increased attention in controlled release technology owing to ease for fabrication and modification to meet special needs. However, there exists an urgent need to design such a matrix system to appropriately regulate the release of therapeutically active agents. The release of solutes from hydrogel networks is strongly influenced by the properties of polymers, geometry of hydrogels and physicochemical properties of encapsulated agents. ${ }^{1}$ Currently, most interest in regulating the release behavior of bioactive compounds from hydrogels arises from the acquirement of macromolecular structure with special functional groups, which respond to changes in environmental conditions. Stimuli-sensitive hydrogels that undergo volume change in response to external stimuli, such as temperature, $\mathrm{pH}$ and/or chemicals have been extensively investigated. ${ }^{2-7}$ The release of solute can, accordingly, be programmed to meet special requirements by exploiting external variation in temperature, $\mathrm{pH}$ or concentrations of chemicals. Many attempts have been made to improve the rate and amplitude of response from hydrogels with respect to performance as controlled release matrices. ${ }^{8,9}$ Yoshida et al. successfully prepared comb-type $\operatorname{poly}(N$ isopropylacrylamide) (PNIPAAm) grafted hydrogels for pulsatile response in deswelling to temperature increase. ${ }^{8}$ Chen and Hoffman reported that the graft copolymerization of PNIPAAm with acrylic acid (AAc) rendered the temperature sensitivity of these copolymers pH-dependent. ${ }^{9}$ By change in temperature, the phase separation of the graft copolymers in aqueous solutions occurs sharply at low $\mathrm{pH}$ regardless of the content of AAc.

Biodegradable hydrogels have become a subject of great interest, especially in applications of site-specific drug delivery. Extensive degradation of hydrogels at desired sites enables the gels to effectively liberate encapsulated agents. We previously prepared a $\mathrm{pH}$-sensitive

\footnotetext{
${ }^{\dagger}$ To whom correspondence should be addressed.
}

dextran hydrogel system for potential colon-specific delivery of therapeutically active proteins or compounds. ${ }^{7}$ By exploiting change in $\mathrm{pH}$ along the gastrointestinal tract, bioactive agents were protected from enzymatic degradation via deswelling of the gel whereas an effective liberation of drugs was achieved through swelling and degradation of dextran hydrogels in colon. Similarly, a hydrogel system developed by Yeh et al. was synthesized by cross-linking of acrylic acid-containing polymeric precursors with an azoaromatic agents. ${ }^{10}$ The hydrogels became microbically degradable by reductases as extensive swelling occurred during the residence of the gel in colon. Chujo et al. developed a hydrogel system capable of undergoing reversible gel disintegration and re-formation in response to reduction-oxidation reaction. ${ }^{11,12}$ Hydrogels prepared from either redox reaction of thio-protected poly( $N$-acetylethylenimine) (PAEI) or cross-linking of partially hydrolyzed PAEI with bifunctional disulfide compounds exhibited reversibility in change in polymer phases between hydrogels and soluble polymers by interconversion of disulfide and sulfhydryl groups. ${ }^{11}$ The behavior of thermally and redox reversible conversion between soluble polymers and hydrogels was observed from a cobalt (III) bipyridylbranched PAEI complex system as compared to only thermal-induced phase changes from the iron (II) complexes. ${ }^{12,13}$

Various strategies for preparing hydrogels have been used. Copolymerization of low molecular weight monomers containing bi- and tetra-functionalities has been the most frequently employed, in which the latter acts as cross-linkers during gel formation. However, the average molecular weights and compositions of copolymers, especially in cases that more than two comonomers were used, were rather difficult to be determined. Recently, van Dijk et al. prepared dextran hydrogels from free radical polymerization of high molecular weight glycidyl methacrylate derivatized dextran. ${ }^{14}$ The contents of methacrylate in dextran polymers and hydrogels were 
Table I. Molar ratios of monomers in copolymerization reactions and characterization of polymeric precursors

\begin{tabular}{ccccc}
\hline \multirow{2}{*}{ Polymer ID } & $\begin{array}{c}\text { Molar ratios in the reaction feeds }^{\mathrm{a}} \\
\text { AAc/BA/NIPAAm/MAGlyGlyONp }\end{array}$ & $\begin{array}{c}\text { Compositions of polymeric precursors }{ }^{\mathrm{b}} \\
\text { AAc/BA/NIPAAm/MAGlyGlyONp/mol\% }\end{array}$ & $M_{w} / \mathrm{g} \mathrm{mol}^{-1}$ c $^{2}$ Polydispersity $^{2}$ \\
\hline A & $10 / 23 / 55 / 12$ & $6.9 / 16.4 / 72.2 / 4.5$ & 21600 & 2.2 \\
B & $15 / 18 / 55 / 12$ & $10.1 / 4.4 / 82.2 / 3.3$ & 17600 & 2.5 \\
C & $5 / 28 / 55 / 12$ & $2.7 / 18.7 / 75.0 / 3.6$ & 15200 & 2.7 \\
D & $15 / 28 / 45 / 12$ & $9.1 / 29.9 / 57.1 / 3.9$ & 11300 & 2.1 \\
E & $10 / 13 / 65 / 12$ & $8.4 / 10.4 / 76.5 / 4.7$ & 13000 & 2.0 \\
F & $15 / 24 / 45 / 16$ & $11.2 / 8.6 / 75.7 / 4.5$ & 8600 & 1.9 \\
\hline
\end{tabular}

${ }^{\mathrm{a}}$ Monomers : AIBN : DMF $=12.5: 0.6: 86.9(\mathrm{wt} \%) .{ }^{\mathrm{b}}$ Determined by ${ }^{1} \mathrm{H}$ NMR and titration. ${ }^{\mathrm{c}}$ Based on weight-average molecular weight as determined by GPC.

well characterized by nuclear magnetic resonance (NMR) and Fourier transform infrared (FT-IR) spectrocsopies. Preparation of inulin hydrogels was reported in a similar manner. ${ }^{15}$ Another approach for preparing hydrogels involves the synthesis of polymeric precursors containing reactive function groups, followed by crosslinking reactions between the reactive groups with appropriate cross-linkers. Characterization of polymeric precursors may give better control of properties and, consequently, biological performance of hydrogels.

The present study develops a functional hydrogel system as potential stimuli-responsive drug delivery carriers using a bifunctional disulfide agent for cross-linking of $\mathrm{pH} /$ temperature-sensitive polymers. Polymeric precursors comprising $N$-isopropylacrylamide (NIPAAm), $N$ - $t$-butylacrylamide (BA), AAc and $N$-methacryloylglycylglycine $p$-nitrophenylester (MAGlyGlyONp) were prepared by free radical polymerization and their compositions and average molecular weights were characterized. The temperature at which the phase separation of the copolymers in aqueous phase occurred was also determined. Preparation of double stimuli-sensitive and disulfide linkage-containing hydrogels was performed from cross-linking of polymeric precursors with cystamine. Characterization of hydrogels with respect to degree of equilibrium swelling and swelling reversibility as functions of temperature and $\mathrm{pH}$ was carried out. The effective network density of hydrogels was estimated by mechanical measurement. The extent of chemical degradation of hydrogels by low molecular weight sulfhydrylcontaining compounds at different $\mathrm{pH}$ was studied. The release of bovine serum albumin (BSA) as a protein drug model from hydrogels is described.

\section{EXPERIMENTAL}

\section{Materials}

NIPAAm, BA, AAc, methacryloyl chloride (MACl), and glycylglycine (GlyGly) were obtained from Acros, NJ, USA. NIPAAm and BA were purified by recrystallization twice from hexane/cyclohexane $(1: 1 \mathrm{v} / \mathrm{v})$ and acetone, respectively. AAc was distilled under reduced pressure before use. 2,2'-Dithiobis(ethylamine) dihydrochloride (cystamine dihydrochloride), glutathione (reduced form), 2-mercaptoethanol, L-cysteine, dithioerythritol (DTT), $p$-nitrophenol, $N, N^{\prime}$-dicyclohexylcarbodiimide (DCC) were purchased from TCI, Tokyo, Japan. 4-Methylmorpholine and 2,2'-azobisisobutyronitrile (AIBN) were obtained from Aldrich, WI, USA. AIBN was purified by recrystallization from methanol twice before use. BSA and 2,4,6-trinitrobenzene sulfonic acid (TNBS) were purchased from Sigma, MI, and Bio-Rad (Coomassie blue) protein assay agent obtained from Bio-Rad, CA, USA. All other chemicals were analytic grade and used as received. Preparation of MAGlyGlyONp was carried out as described elsewhere. ${ }^{16}$ In brief, Schotten-Baumann reaction of fresh distilled $\mathrm{MACl}$ with glycylglycine in $4 \mathrm{~N}$ $\mathrm{NaOH}$ gave MAGlyGly (mp: $190^{\circ} \mathrm{C}$, yield $95 \%$ ), followed by reaction of this methacryloyl dipeptide with $p$ nitrophenol in dimethylformamide (DMF) at $-10^{\circ} \mathrm{C}$ using DCC as the coupling agent. MAGlyGlyONp was obtained in $75 \%$ yield (mp: $163-166^{\circ} \mathrm{C} ; \varepsilon=9900 \mathrm{M}^{-1} \mathrm{~cm}^{-1}$ (DMSO); thin layer chromatography (TLC): $R_{\mathrm{f}}=0.195$ (ethyl acetate), single spot).

\section{Synthesis of Polymeric Precursors}

Polymeric precursors were prepared by semi-batch free radical polymerization of NIPAAm, BA, AAc, and MAGlyGlyONp using AIBN as initiator. Polymerization was carried out in DMF solution containing $12.5 \mathrm{wt} \%$ of monomers and $0.6 \mathrm{wt} \%$ of AIBN at $60^{\circ} \mathrm{C}$ with stirring under $\mathrm{N}_{2}$ for $20 \mathrm{~h}$. NIPAAm was added in DMF and polymerization proceeded for 15 min prior to the addition of AAc and BA in DMF solution. ${ }^{17}$ After polymerization, the volume of the reaction solution was reduced to $c a$. $30 \%$ using a rotary evaporator under reduced pressure. The copolymer was precipitated from ethyl ether/ethyl acetate $(2: 1 ; \mathrm{v} / \mathrm{v})$ three times. The polymer was filtered and washed by precooled ether/ethyl acetate and dried in vacuo. The complete removal of residual unreacted monomer (especially, unreacted MAGlyGlyONp monomer) and/or by-products was confirmed by TLC, gel permeation chromatography (GPC) (Pharmacia FPLC, Superose 12 column) and FT-IR (Beckman Paragon 500). Mole ratios of monomers in the reaction feeds for preparation of various copolymers are listed in Table I.

\section{Characterization of Polymeric Precursors}

The compositions of polymeric precursors were mainly characterized by ${ }^{1} \mathrm{H}$ NMR (Varian VXR, $300 \mathrm{MHz}$ ). The samples were run in DMSO- $d_{6}$ at ambient temperature and integrated signals corresponding to protons from different comonomers were compared. Absolute content (mol $\mathrm{g}^{-1}$ ) of MAGlyGlyONp in copolymers was determined spectrometrically in DMSO $(274 \mathrm{~nm}, \varepsilon=9600$ $\left.\mathrm{M}^{-1} \mathrm{~cm}^{-1}\right) .{ }^{18}$ Owing to possible occurrence of ionization from AAc comonomer in DMSO- $d_{6}$ that might lead to mis-calculation of AAc content from NMR characterization, the copolymers (100 mg each) were subjected to aminolysis with 3-aminopropanol and content of AAc was determined by titration (Mettler DL-53) with $0.01 \mathrm{M}$ $\mathrm{NaOH}$. Average molecular weights of copolymers were 
Table II. Parameters for preparing hydrogels

\begin{tabular}{cccc}
\hline Hydrogel ID & Polymer ID & $\begin{array}{c}\text { Polymer } \\
\text { conc/wt\% }\end{array}$ & $\begin{array}{c}\text { Cystamine } \\
\text { conc./mmol g }\end{array}$ \\
\hline H1 & A & 30 & 0.36 \\
H2 & B & 30 & 0.36 \\
H3 & C & 30 & 0.36 \\
H4 & D & 30 & 0.29 \\
H5 & D & 30 & 0.36 \\
H6 & E & 30 & 0.36 \\
H7 & F & 30 & 0.40 \\
H8 & F & 40 & 0.40 \\
\hline
\end{tabular}

${ }^{a}$ Concentration of cross-linking agent expressed as mmol per gram of polymeric precursor.

estimated by GPC (Pharmacia, FPLC Superose 12) using Tris buffer ( $\mathrm{pH} 7.4,0.1 \mathrm{M}$ with $0.1 \mathrm{M} \mathrm{NaCl}$ ) as eluent (flow rate: $0.4 \mathrm{~mL} \mathrm{~min}{ }^{-1}$ ) under refractometric (Jasco RI-930) and UV/Vis detection (Pharmacia, UV-1). Poly( $N$-2-hydroxypropylmethacrylamide) fractions were used as standards for molecular weight calibration.

Cloud points of copolymers were evaluated by measuring temperatures at which $10 \%$ reduction in transmittance at $500 \mathrm{~nm}$ from copolymer aqueous solutions $(2.0$ mg mL ${ }^{-1}$; phosphate buffer, $\mathrm{pH} 7.4$ ) was observed spectrometrically. ${ }^{9}$

\section{Hydrogel Preparation}

Hydrogels were prepared from cross-linking of polymeric precursors with cystamine dihydrochloride as cross-linking agent. To a solution of polymeric precursor $(30 \mathrm{wt} \%)$ in DMSO, a selected amount of cystamine dihydrochloride (according to - ONp content in the copolymer and desired degree of chemical cross-linking) was added. After stirring for $2 \mathrm{~min}$, 4-methylmorpholine $(0.2 \mathrm{~mL}$ for all hydrogel preparations) was subsequently added and the reaction solution was vortexed for $30 \mathrm{~s}$. After $24 \mathrm{~h}$ at room temperature, the hydrogel was carefully taken out and thoroughly washed with ethanol and deionized water. Hydrogels prepared in this study are listed in Table II. Hydrogel $\mathrm{H} 8$ was prepared from a $40 \mathrm{wt} \%$ solution of the corresponding polymer in DMSO.

\section{Hydrogel Swelling Measurements}

Hydrogels were dried in vacuo at $55^{\circ} \mathrm{C}$ for $72 \mathrm{~h}$. The pre-weighed dry gels were placed in various $\mathrm{pH}$ buffer

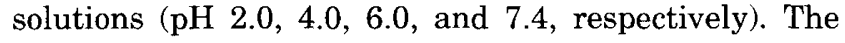
ionic strength of all buffer solutions was adjusted to 0.1 with $\mathrm{NaCl}$. The experiment was performed for $72 \mathrm{~h}$ to ensure equilibrium swelling. Swelling is expressed in terms of swelling ratio, defined as ${ }^{19}$

$$
\text { Swelling ratio }=\left(W_{\mathrm{g}}-W_{\mathrm{d}}\right) / W_{\mathrm{d}}
$$

where $W_{\mathrm{g}}$ and $W_{\mathrm{d}}$ are the weights of the swollen and dry gel, respectively. Equilibrium swelling of hydrogels as a function of temperature was also carried out by measuring swelling ratios of hydrogels at $25,37,40$, and $50^{\circ} \mathrm{C}$ in pH 2.0 and 7.4 buffer solutions for $72 \mathrm{~h}$, respectively.

For study of swelling reversibility in response to repeated change in $\mathrm{pH}$, dry hydrogels were placed in $\mathrm{pH}$ 5.0 buffers and swelling ratios were determined at various time intervals. The hydrogels were withdrawn and placed in $\mathrm{pH} 4.0$ buffer solution. The extent of gel hydra- tion as a function of time was measured again and the procedure was repeated. Swelling reversibility of hydrogels induced by change in temperature between 25 and $40^{\circ} \mathrm{C}$ was performed in $\mathrm{pH} 2.0$ and 7.4 buffer solutions, respectively.

Determination of Effective Network Density of Hydrogels

The effective network density of hydrogels was determined by measuring the modulus of elasticity in compression as described elsewhere. ${ }^{20,21}$ The hydrogels were placed in a $\mathrm{pH} 7.4$ buffer solution at $25^{\circ} \mathrm{C}$ for $72 \mathrm{~h}$ and, subsequently, cut into $1 \mathrm{~cm}$-in-diameter pieces. Equilibrium heights at various compressive stress were determined at the same temperature using a bench comparator (Ames 135). The modulus of elasticity at equilibrium was obtained according to the equation ${ }^{21}$

$$
F / A=-G\left(\lambda-\lambda^{-2}\right)
$$

where $F / A$ is the compressive stress applied, $G$ the equilibrium modulus of elasticity and $\lambda=l l_{0}$ (where $l_{0}$ and $l$ are the heights of original and deformed gels). The modulus of elasticity $(G)$ was obtained from the slope of the linear plot of $F / A$ versus $-\left(\lambda-\lambda^{-2}\right)$. The effective network density was calculated from the equation ${ }^{21}$

$$
v_{\mathrm{e}}=G /\left(R T v_{2, \mathrm{~s}}{ }^{1 / 3}<\alpha>^{2}\right)
$$

where $v_{\mathrm{e}}$ is the concentration of elastically effective

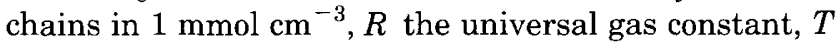
the absolute temperature, $v_{2, \mathrm{~s}}$ polymer volume fraction of the swollen gel, and $\langle\alpha\rangle^{2}=v_{0}{ }^{2 / 3}$ (where $v_{0}$ is the polymer volume fraction of hydrogel in the relaxed state) according to ref 22 . The densities of polymers were determined pycnometrically and an average value of 1.35 $( \pm 0.02) \mathrm{g} \mathrm{cm}^{-3}$ was used for all polymers in calculation.

\section{Release Measurement of Bovine Serum Albumin (BSA) from Hydrogels}

To a buffer solution ( $20 \mathrm{~mL}$; pH 7.4 ) of BSA (40 mg $\mathrm{mL}^{-1}$ ), a dried gel was immersed. The hydrogel was taken out and washed with distilled water after equilibrating in the BSA concentration for 5 days. The loading amount of protein into hydrogels was determined by measuring differences in BSA concentrations in solutions spectrometrically. BSA-loaded hydrogels were washed with distilled water and placed in $\mathrm{pH} 2.0$ and 7.4 buffer solutions with ionic strength adjusted to 0.1 , respectively. At preset time intervals, the medium $(0.1$ $\mathrm{mL}$ ) was withdrawn and replaced with fresh buffer. Aliquots were analyzed for BSA concentrations via Bradford protein assay. The experiment was performed in duplicate.

\section{Degradation Measurement of Hydrogels}

To a solution of 2-mercaptoethanol $(0.014 \mathrm{M})$ in $15 \mathrm{~mL}$ of $\mathrm{pH} 7.4$ buffer solution, a pre-weighed dry gel was immersed. The reaction was carried out at $37^{\circ} \mathrm{C}$ in a constant temperature water bath. Hydrogels were withdrawn at various times and thoroughly washed with ethanol and deionized water and dried in vacuo for $24 \mathrm{~h}$. The degraded gels were weighed and extent of degradation was determined as percentage of weight loss of dry 


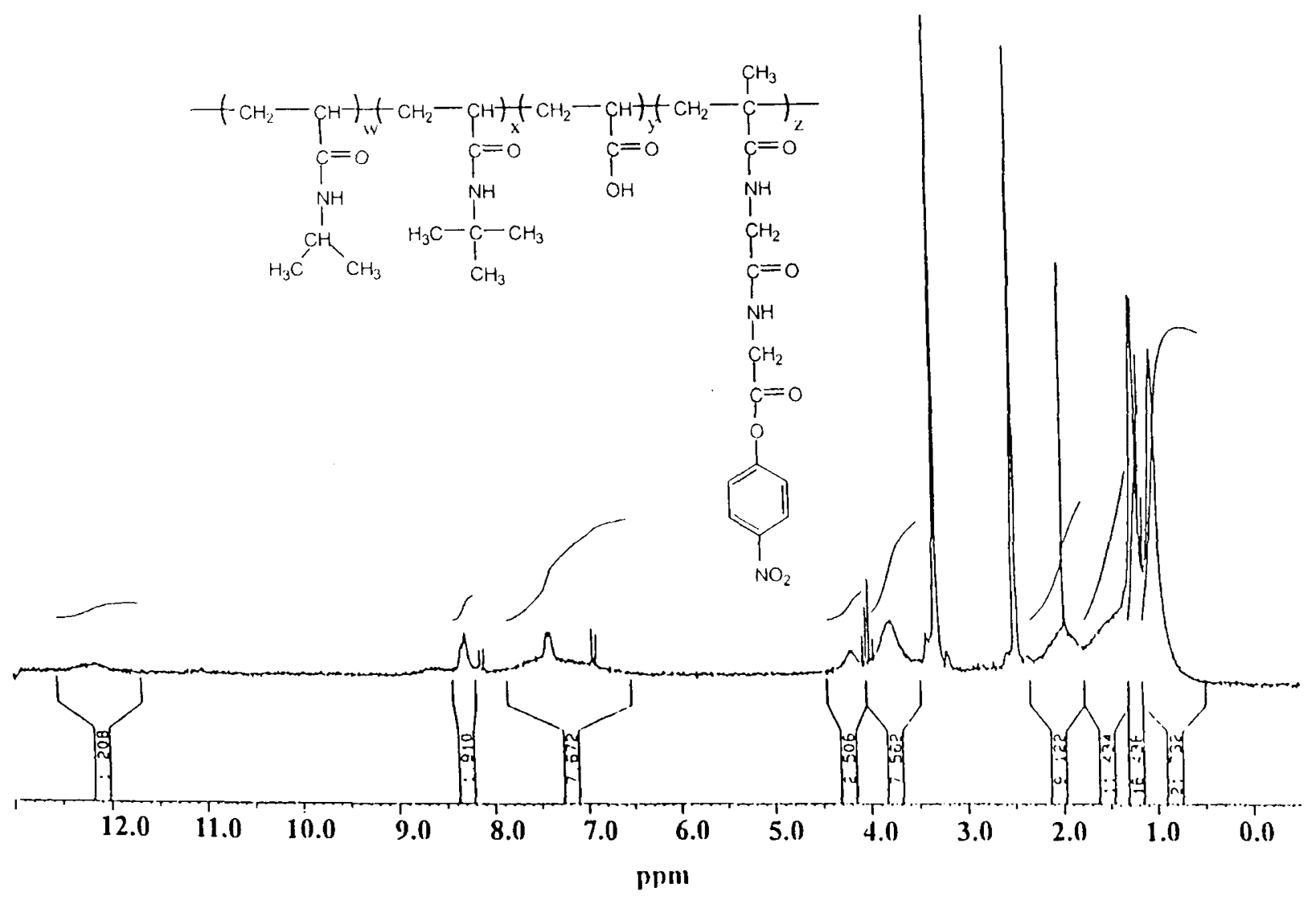

Figure 1. Chemical structure of polymeric precursors and ${ }^{1} \mathrm{H}$ NMR spectrum of copolymer $\mathrm{F}$.

gels. The procedures were repeated while the buffer solution was changed to $\mathrm{pH} 2.0$ hydrochloride aqueous solution. Disulfide reducing agents, L-cysteine, DTT and glutathione (reduced form) were also used $(0.014 \mathrm{M})$ for the degradation of hydrogels in $\mathrm{pH} 7.4$ buffer solution at $37^{\circ} \mathrm{C}$ and the extent of degradation was measured. Extensive degradation of hydrogels by cysteine $(0.1 \mathrm{M})$ was also performed for $48 \mathrm{~h}$ at $37^{\circ} \mathrm{C}$. The reaction mixture was subjected to dialysis against distilled water for 5 days and lyophilization. Molecular weights of the collected polymers and amino content were characterized by GPC (Pharmacia, FPLC Superose 12) and TNBS measurements, respectively. ${ }^{23}$

\section{RESULTS AND DISCUSSION}

Synthesis of temperature/pH-sensitive hydrogels has been reported elsewhere. ${ }^{3-6,17,19}$ For example, Park prepared double stimuli-responsive hydrogels from copolymerization of NIPAAm, $N, N$-dimethylaminopropylacrylamide and $N, N^{\prime}$-methylene-bis-acrylamide. ${ }^{4}$ In this study, temperature/pH-sensitive hydrogels were synthesized by first preparing polymeric precursors containing NIPAAm, AAc, BA, and MAGlyGlyONp, followed by aminolysis of the precursors with cystamine, a disulfidecontaining cross-linker. Polymerization was carried out in a semi-batch manner as described previously. Polymeric precursor was purified by precipitation from ethyl ether/ethyl acetate solution three times. The complete removal of unreacted monomers and/or low molecular weight by-products was confirmed by TLC and GPC.
Characterization of polymeric precursors by FT-IR indicated the absence of a peak at $c a .1605-1635 \mathrm{~cm}^{-1}$ attributed to $\mathrm{C}=\mathrm{C}$ whereas typical peaks for $\mathrm{C}-\mathrm{H}$ stretching in aromatic ring (ca. $\left.3100 \mathrm{~cm}^{-1}\right), \mathrm{N}-\mathrm{H}$ bending from NIPAAm and BA (ca. $\left.1640 \mathrm{~cm}^{-1}\right)$ and $\mathrm{O}-\mathrm{H}$ bending from AAc $\left(\mathrm{ca} .930 \mathrm{~cm}^{-1}\right)$ were observed.

The compositions of polymeric precursors were primarily determined by ${ }^{1} \mathrm{H}$ NMR spectra. Figure 1 shows the NMR spectrum of copolymer F. The integrated area obtained from the signal at $8.3 \mathrm{ppm}$ was attributed to $-\mathrm{C}_{6} \mathrm{H}_{4}$ - of MAGlyGlyONp whereas the area between 3.7 and $4.3 \mathrm{ppm}$ was assigned to two methylene groups from -GlyGly- of MAGlyGlyONp and - $\mathrm{CH}^{-}$of NIPAAm. The composition of BA was obtained from the integrated area between 0.9 to $2.2 \mathrm{ppm}$, which was assigned to one $-\mathrm{CH}_{3}$ group from MAGlyGlyONp, two $-\mathrm{CH}_{3}$ from NIPAAm and three $-\mathrm{CH}_{3}$ from BA. Since partial ionization of AAc might occur in DMSO- $d_{6}$, the relative composition ratios of AAc were obtained by comparing absolute content of AAc and MAGlyGlyONp. The content of AAc was determined by titration of polymeric precursors in which the MAGlyGlyONp comonomer was first aminolyzed with 3aminopropanol while the absolute content of MAGlyGlyONp was evaluated spectrometrically (DMSO, 274 $\mathrm{nm}, \varepsilon=9600 \mathrm{M}^{-1} \mathrm{~cm}^{-1}$ ). The compositions of polymeric precursors are listed in Table I. From Table I, NIPAAm possesses a higher reactivity ratio than other three comonomers during copolymerization. The significantly lower content of MAGlyGlyONp in copolymers and lower value for the average molecular weight of copolymer $F$ were probably related to the chain-transfer action of the 


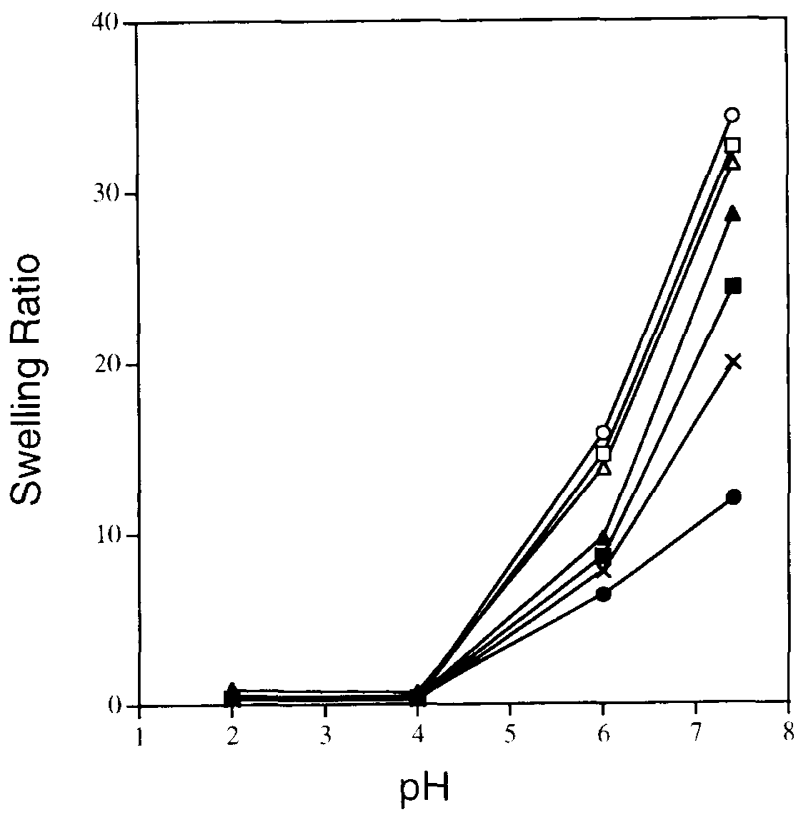

Figure 2. Equilibrium swelling of hydrogels $\mathrm{H} 1$ ( $\square$ ), H2 ( $\square$ ), H3 $(\odot), \mathrm{H} 4(\bigcirc), \mathrm{H} 5(\Delta), \mathrm{H} 7(\triangle)$, and $\mathrm{H} 8(\times)$ as a function of $\mathrm{pH}$.

comonomer (Table I). ${ }^{10}$ Average molecular weights and molecular weight distribution of polymeric precursors were evaluated by GPC using fractionated poly $(\mathrm{N}-2$ hydroxypropylmethacrylamide) as the molecular weight calibration standard and the results are shown in Table I.

The extent of equilibrium swelling of hydrogels as a function of $\mathrm{pH}$ and of temperature is shown in Figures 2 and 3 , respectively. No phase separation of macromolecules was observed spectrometrically as hydrogels reached the equilibrium swelling. While, at $\mathrm{pH}$ above 6.0 , equilibrium swelling was strongly influenced by AAc content of hydrogels, swelling extent was rather reduced at low $\mathrm{pH}$ and differences became insignificant irrespective of AAc content (Figure 2). It is generally believed that ionization of AAc in gels at $\mathrm{pH}$ higher than gel $\mathrm{pKa}$ leads to increase in hydrophilicity and charge repulsion and, consequently, elevation of water uptake. ${ }^{24}$ Hydrogels $\mathrm{H} 1, \mathrm{H} 2$, and $\mathrm{H} 3$ were prepared from the same polymer concentrations and comparable chemical crosslinking. However, hydrogel H2 exhibited higher equilibrium swelling at high $\mathrm{pH}$ than $\mathrm{H} 1$ and $\mathrm{H} 3$ mostly owing to higher content of AAc and lower BA. The lowest swelling at $\mathrm{pH} 7.4$ was observed from hydrogel $\mathrm{H} 3$ as a consequence of its lowest content of AAc and somewhat higher content of BA.

Equilibrium swelling was significantly affected by the concentrations of copolymer and cross-linking agent in the preparation of hydrogels. Hydrogels $\mathrm{H} 4$ and H5 prepared from copolymer $\mathrm{D}$ at the same polymer concentration, but with different degrees of chemical cross-linking exhibited distinct swelling at higher $\mathrm{pH}$. The highest equilibrium swelling was observed from gel $\mathrm{H} 4$ in response to $\mathrm{pH}$ 7.4. Swelling of H5 was significantly reduced. Hydrogels with higher molecular weight between cross-links (i.e., lower degree of chemical cross-linking) provided higher flexibility for extending their polymer chains during hydration. Rather low equilibrium swel-

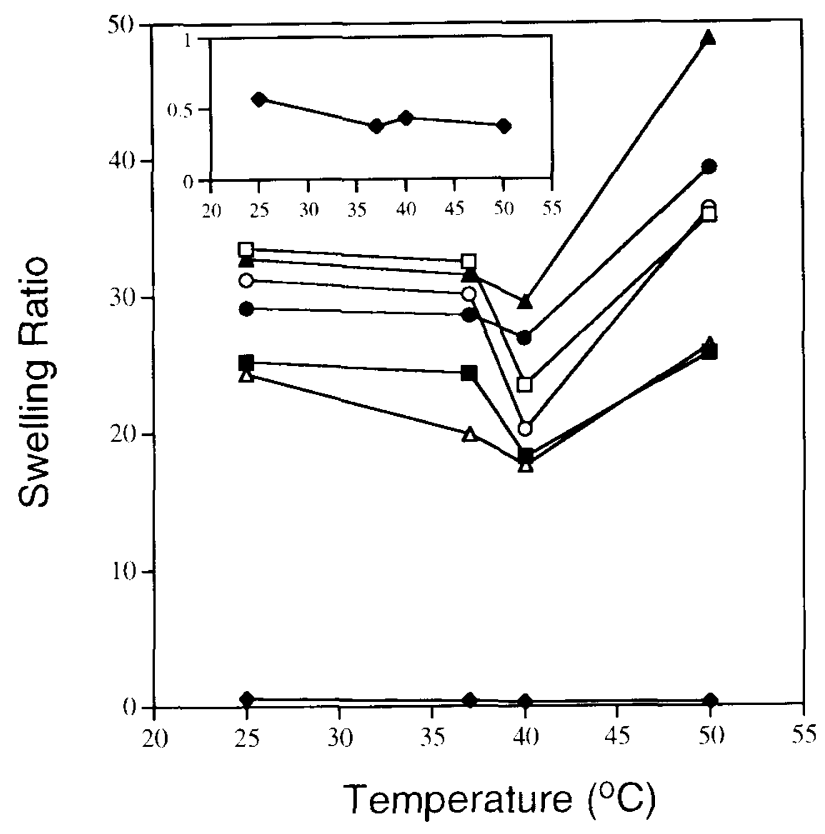

Figure 3. Equilibrium swelling of hydrogels H1 ( $\square$ ), H2 ( $\square$ ), H5 ( ), $\mathrm{H} 6\left({ }_{(}\right), \mathrm{H} 7(\Delta)$, and $\mathrm{H} 8(\triangle)$ at $\mathrm{pH} 7.4$ and $\mathrm{H} 2(\bullet)$ at $\mathrm{pH} 2.0$ as a function of temperature.

ling was observed from hydrogel $\mathrm{H} 8$ at $\mathrm{pH} 6.0$ and 7.4 in spite of its highest content of AAc. This was attributed mostly to the high concentration ( $40 \mathrm{wt} \%$ ) of polymer as well as elevated chemical cross-linking density $(0.4$ mmol $\mathrm{g}^{-1}$ of polymer). The effect of polymer concentration on equilibrium swelling became even more evident as swelling between hydrogels $\mathrm{H} 7$ and $\mathrm{H} 8$ was compared at high $\mathrm{pH}$. It is believed that increasing polymer concentration during gel formation inevitably results in increase in physical entanglement of polymer chains and restricts molecular mobility. ${ }^{25}$ Insignificant differences in equilibrium swelling at low $\mathrm{pH}$ (from 0.4 to 0.8 ) were attributed to the hydrophilic nature of the polymeric precursors, probably owing to the general low content of BA. Little effect of molecular weights of polymers on gel hydration was observed. It is generally believed that the intrinsic entanglement of polymer chains should reduce with decrease in molecular weight of polymer. ${ }^{26}$ Nevertheless, hydrogel H8, which possesses the lowest average molecular weight, demonstrated relatively low equilibrium swelling owing to high concentrations of polymer and cross-linking agent.

The dependence of equilibrium swelling on temperature is illustrated in Figure 3. At pH 7.4, swelling was reduced in response to change in temperature from 37 to $40^{\circ} \mathrm{C}$. These results are approximately in agreement with the cloud points of the precursors (from 38.5 to 42.5 ${ }^{\circ} \mathrm{C}$ ) measured at $10 \%$ reduction in transmittance in $\mathrm{pH}$ 7.4 buffer solutions. NIPAAm-containing (co)polymers in aqueous phases undergo phase separation by temperature increase as primarily a result of enhanced aggregation of hydrophobic residues and breakage of hydrogen bonding with water. ${ }^{27,28}$ Consequently, hydrogels undergo volume change in response to temperature variation in the range that the phase separation of polymers occurs. However, reduction in swelling in response to temperature stimulus was less pronounced than that by 


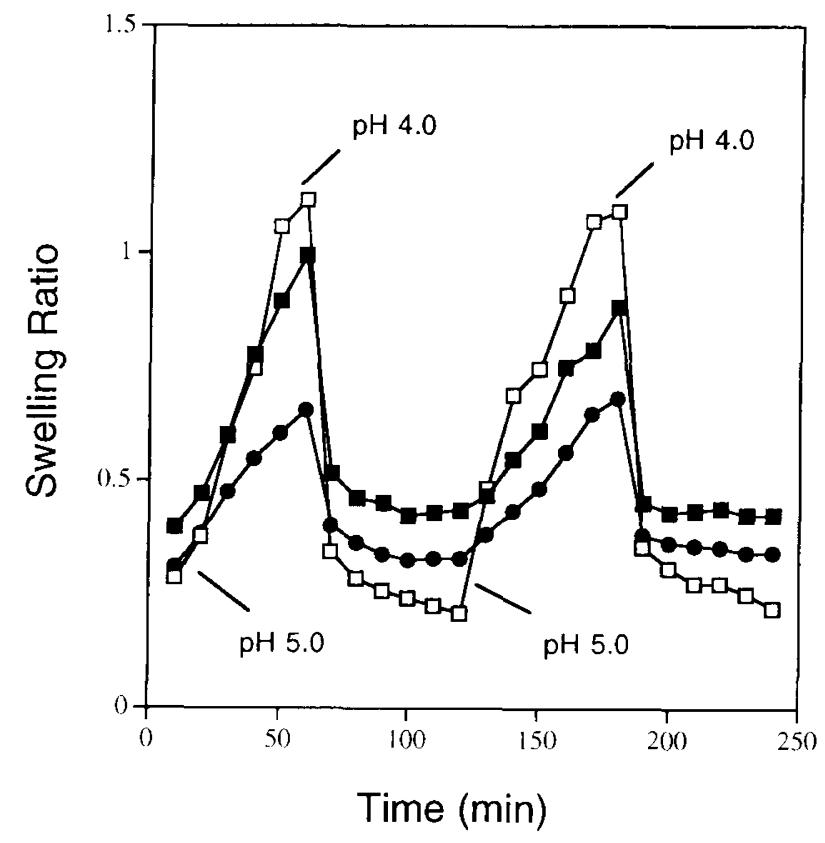

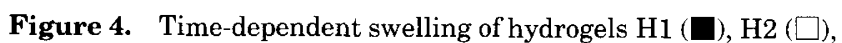
and $\mathrm{H} 3(\mathrm{)})$ in response to repeated abrupt change in $\mathrm{pH}$ between 4.0 and 5.0 .

change in $\mathrm{pH}$. Yoshida et al. suggest that a low degree of gel dehydration results from formation of a dense, water-impermeable polymer layer near the gel surface. ${ }^{29}$ The layer formation retarded the release of entrapped bulk water from collapsing gels. Equilibrium swelling of hydrogels increased at $50^{\circ} \mathrm{C}$ in pH 7.4 buffer solutions. This, although somewhat surprising, is consistent with the observation reported by Dong and Hoffman, ${ }^{30}$ probably owing to increased ionization of AAc under $50^{\circ} \mathrm{C}$ and $\mathrm{pH}$ 7.4. The decrease in deswelling from hydrogels $\mathrm{H} 5, \mathrm{H} 7$, and $\mathrm{H} 8$ was mostly related to lower content of NIPAAm and/or higher cross-linking density. Temperature-induced change in gel volume at $\mathrm{pH} 2.0$ was relatively low compared to that in $\mathrm{pH} 7.4$ buffers owing to significantly reduced equilibrium swelling under $\mathrm{pH} 2.0$ conditions. Reduction in gel volume occurred in the temperature range from 25 to $37^{\circ} \mathrm{C}$. This is in agreement with the cloud point measurements of polymeric precursors $\left(c a .30 .5\right.$ to $37.5^{\circ} \mathrm{C}$ ) at $\mathrm{pH} 2.0$. The reduction in the cloud points of precursors is primarily due to increased hydrophobic nature of polymers at $\mathrm{pH} 2.0$. Equilibrium swelling was rather independent of the temperature above $40^{\circ} \mathrm{C}$ in $\mathrm{pH} 2.0$ aqueous solutions.

Reversible swelling-deswelling of hydrogels in response to repeated changes in $\mathrm{pH}$ between 4.0 and 5.0 was observed (Figure 4). Hydrogel $\mathrm{H} 2$ exhibited the fastest response in swelling rate and amplitude owing to high content of AAc. The low content of AAc in gel H3 rendered hydration capability more restricted. Dehydration of gel $\mathrm{H} 2$ at first occurred sharply and then gradually decreased whereas shrinkage of gels $\mathrm{H} 1$ and $\mathrm{H} 3$ quickly reached equilibrium state of gel relaxation. The higher water uptake of gels $\mathrm{H} 1$ and $\mathrm{H} 3$ in the relaxed state than that of $\mathrm{H} 2$ was attributed to lower content of un-ionized AAc at $\mathrm{pH}$ 2.0. The higher deswelling rates of hydrogels $\mathrm{H} 1$ and $\mathrm{H} 3$ to reach the equilibrium state were explained by the nature of increased hydrophobic-
Table III. Network parameters for hydrogels

\begin{tabular}{ccrc}
\hline Hydrogel ID & $v_{2, \mathrm{~s}}^{\mathrm{a}}$ & $G_{0} / \mathrm{Pa}$ & $v_{\mathrm{e}} / \mathrm{mmol} \mathrm{cm}^{-3}$ \\
\hline H1 & 0.028 & 12300 & 0.040 \\
H2 & 0.022 & 4800 & 0.017 \\
H3 & 0.045 & 18700 & 0.052 \\
H4 & 0.024 & 4100 & 0.014 \\
H5 & 0.025 & 12600 & 0.043 \\
H6 & 0.023 & 10100 & 0.035 \\
H7 & 0.022 & $\mathrm{ND}^{\mathrm{b}}$ & $\mathrm{ND}$ \\
H8 & 0.030 & 20600 & 0.066 \\
\hline
\end{tabular}

${ }^{a}$ Polymer volume fraction of hydrogels at equilibrium swelling at $25^{\circ} \mathrm{C}$. ${ }^{\mathrm{b}}$ Not determined.

ity of polymers. Similar observation was reported by several authors in the study of ionic gel systems. ${ }^{19,24}$

The modulus of elasticity and effective network density of hydrogels were estimated according to the theory of rubber-like elasticity ${ }^{20}$ and the results are shown in Table III. Hydrogel H8 showed the highest cross-linking density owing to higher concentration of cystamine in preparation of the hydrogel. The higher concentration of polymeric precursor may also give rise to enhanced physical entanglement of polymer chains. It was reported that the influence of polymer entanglement on crosslinking density was minimized by mechanical measurements. ${ }^{31}$ However, our previous paper pointed out that the effective cross-linking density of ionic hydrogels as evaluated by mechanical measurements varied strongly with the equilibrium degree of swelling at the same initial polymer concentration and comparable chemical cross-linking. ${ }^{7}$ Similarly, different degrees of polymerpolymer and polymer-solvent interaction might lead to variation in cross-linking density. Hydrogels $\mathrm{H} 1, \mathrm{H} 2$, and $\mathrm{H} 3$ were prepared from the same concentrations of polymer and cross-linking agent. The high content of AAc and NIPAAm and low content of BA in hydrogel H2 result in a high degree of equilibrium swelling, which, in turn, decreases the modulus of elasticity and effective cross-linking density. In a similar manner, Bronsted and Kopecek observed that the degree of cross-linking of ionic gels decreased as gels were placed from $\mathrm{pH} 2.0$ to 7.4 buffers. $^{32}$ Ionization of AAc and consequently increased hydrophilicity at $\mathrm{pH} 7.4$ reduce the formation of intra- and inter-macromolecular hydrogen bonding and hydrophobic interaction. The low effective network density of hydrogel $\mathrm{H} 4$ was mainly attributed to the low concentration of cystamine dihydrochloride. From Table III, it becomes evident that the equilibrium degree of swelling decreases with increasing the effective cross-linking density, especially at high $\mathrm{pH}$. As expected, the chemical cross-linking and physical entanglement of polymers restrict the extension capability of polymer chains. The effect of cross-linking density on equilibrium swelling was reduced at $\mathrm{pH} 2.0$ mostly owing to decreased hydrophilicity of the polymers.

Degradation of hydrogels by disulfide reducing agents was performed at $37^{\circ} \mathrm{C}$ in $\mathrm{pH} 7.4$ and 2.0 buffer solutions. Degradation of hydrogels containing disulfide linkages as cross-links have been carried out by Chujo et $a l .{ }^{11}$ In the presence of excess reducing agents, such as sodium borohydride, triphenylphosphine or sodium hydrosulfite, disulfide-bridged PAEI hydrogels were cleaved to linear polymers within $24 \mathrm{~h}$. They also observed that the swelling of PAEI gels increased with partial disul- 


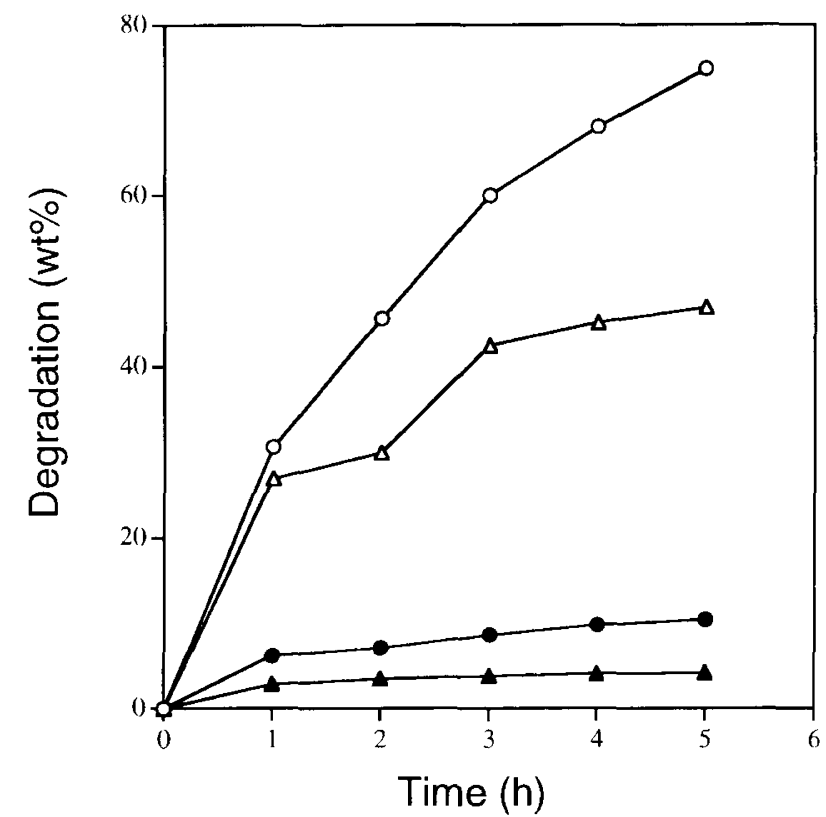

Figure 5. Degradation of hydrogels $\mathrm{H} 4$ (circle) and H5 (triangle) by 2 -mercaptoethanol in $\mathrm{pH} 7.4$ (empty symbols) and 2.0 (solid symbols) buffers at $37^{\circ} \mathrm{C}$ as function of time. The degree of degradation is expressed as reduction percentage in weight of dry gels.

fide cleavage of hydrogels. ${ }^{11}$ In this study, the degradability of hydrogels $\mathrm{H} 4$ and $\mathrm{H} 5$ by 2 -mercaptoethanol at $\mathrm{pH} 7.4$ and 2.0 is shown in Figure 5. Owing to differences in swelling, the degradation of hydrogels occurred more at $\mathrm{pH} 7.4$ than 2.0 , especially after a longer time period of incubation. At $\mathrm{pH} \mathrm{2.0,} \mathrm{low} \mathrm{swelling} \mathrm{retarded}$ the diffusion of 2-mercaptoethanol molecules into gels and degradation occurred mainly at the gel surface. Effective degradation proceeded rather steadily via breakage of disulfide linkages inside gels under $\mathrm{pH} 7.4$ conditions (see the description below). The degradation rate gradually decreased with time mostly due to decrease in the concentration of the cross-links. Differences in swelling between hydrogels $\mathrm{H} 4$ and $\mathrm{H} 5$ resulted in different cleavage rates. Figure 6 shows the degradation of hydrogel $\mathrm{H} 2$ by 2 -mercaptoethanol, L-cysteine and DTT, respectively. Owing to two sulfhydryl (-SH) groups in DTT, the highest degradation rate was observed. Excess reducing agent in the incubation reaction reduced further cross-linking with DTT. The higher degradation rate by L-cysteine than 2-mercaptoethanol was probably due to elevated hydrophilicity and ionic interactions with polymers. The mechanism of bulk degradation at $\mathrm{pH} 7.4$ was substantiated by the observation that intensive yellow color was uniformly displayed throughout the gel after adding TNBS into the L-cysteine-containing reaction mixture. Interestingly, hydrogels after exposure to glutathione (reduced form) exhibited rapid deswelling (Figure 7) and no degradation was observed after $72 \mathrm{~h}$. The reasons are still not clear and further investigation on the mechanism of its response to glutathione and potential applications is in progress. A preliminary study on the degradation of hydrogels by rat cecum content has revealed complete cleavage of gels after $24 \mathrm{~h}$ incubation (data not shown). GPC characterization of linear polymers resulting from extensive degradation of hydrogels by L-cysteine indicated no significant differences in

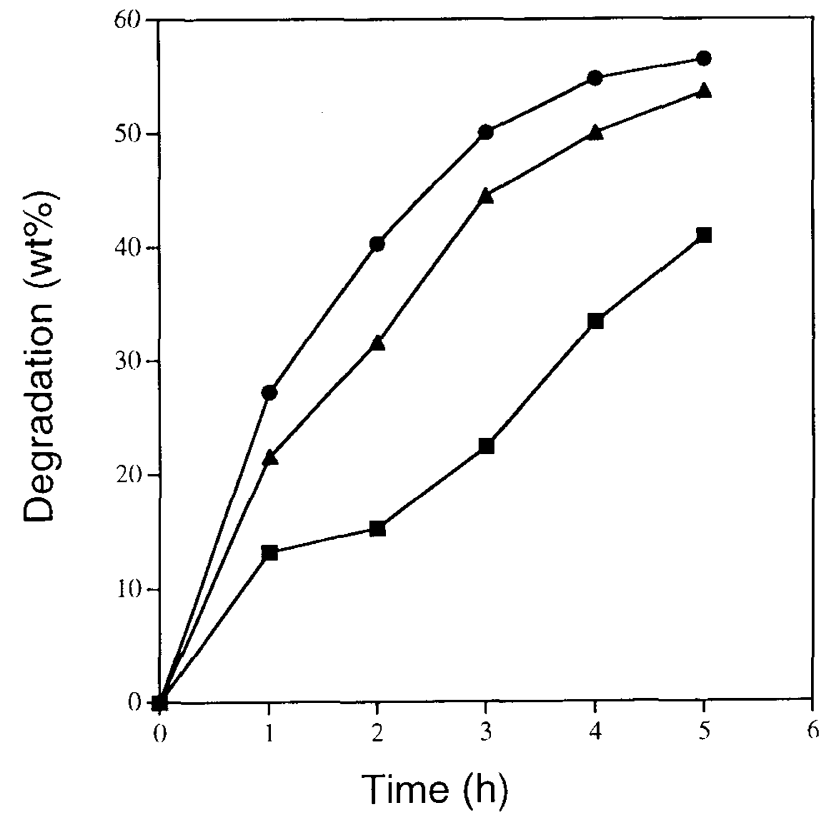

Figure 6. Time-dependent degradation of hydrogel $\mathrm{H} 2$ by 2 mercaptoethanol $(\square)$, L-cysteine $(\mathbf{\Delta})$ and DTT $(\mathbf{O})$ under $\mathrm{pH} 7.4$ and $37^{\circ} \mathrm{C}$ conditions.

molecular weights between polymeric precursors and the resulting polymers after gel degradation. Extensive degradation of hydrogels by L-cysteine produced L-cysteine-containing linear copolymers via disulfide linkages. The amino content of L-cysteine-modified polymers was evaluated by TNBS measurement and the results indicated that 75 to $84 \%$ of the ONp groups of polymeric precursors were conjugated with cystamine. However, the effective network density of hydrogels as determined by the mechanical measurements was much lower mostly owing to significant intramolecular cyclization and crosslinking side reactions. ${ }^{33}$

Hydrogels $\mathrm{H} 1, \mathrm{H} 2, \mathrm{H} 3$, and $\mathrm{H} 8$ were selected for release measurements of BSA (as a macromolecular drug model). The loading amounts of BSA in hydrogels ranged from $c a .5$ to $16 \mathrm{mg}$ per gram of dry gel and loading efficiency in terms of the BSA concentrations in $\mathrm{pH} 7.4$ buffer was ca. $5.2 \%$ (on average). Figure 8 shows the cumulative release of BSA from hydrogels under $\mathrm{pH} 2.0$ and 7.4 conditions. The rate of BSA release was strongly influenced by gel swelling. At pH 7.4, hydrogel $\mathrm{H} 2$ exhibited the highest release rate owing to elevated equilibrium swelling. Differences in release rates of BSA between hydrogels $\mathrm{H} 1$ and $\mathrm{H} 3$ essentially followed those in hydration. BSA release from hydrogel $\mathrm{H} 8$ was more significantly retarded than that from $\mathrm{H} 3$, even though swelling of H8 was higher (Figure 2). Peppas et al. pointed out that the diffusion rate of entrapped agents from hydrogels was primarily determined by mesh size of hydrogels. ${ }^{34}$ Increase in mesh size of hydrogel provides better pathways for solutes to liberate. The equation for mesh size of hydrogel was derived $\mathrm{as}^{34}$

$$
\xi=\left(v_{2, \mathrm{~s}}\right)^{-1 / 3} \times\left(r_{\mathrm{o}}\right)^{1 / 2}
$$

where $\xi$ represents mesh size in $\mathrm{nm}$ and $r_{\mathrm{o}}$ is the average distance between two adjacent cross-links in the solvent-free state. The value of $r_{0}$ strongly depends on 


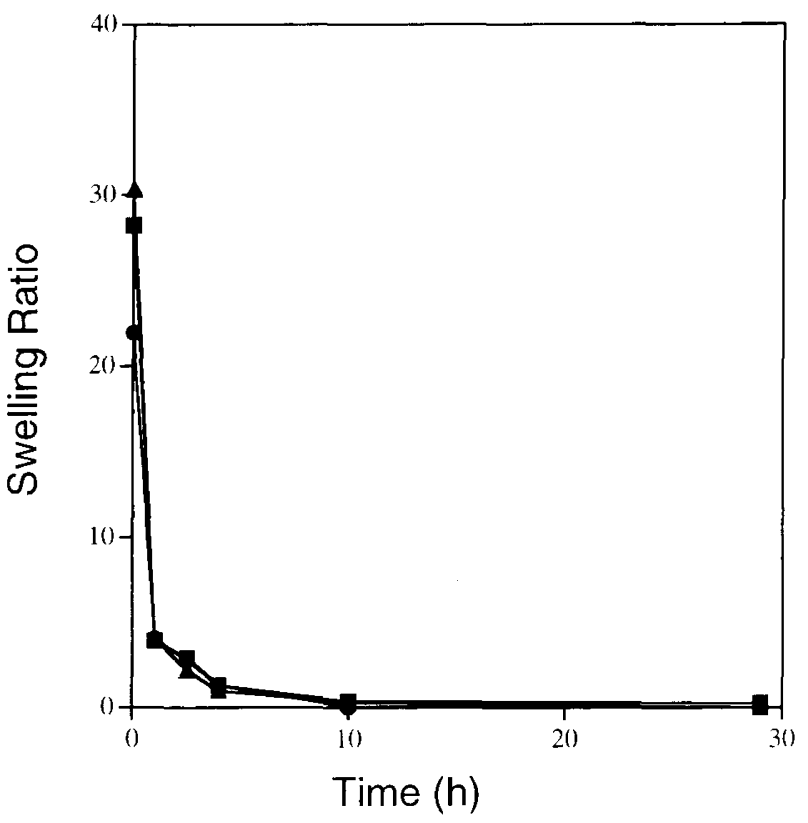

Figure 7. Deswelling of hydrogels $\mathrm{H} 2(\boldsymbol{\square}), \mathrm{H} 4(\boldsymbol{\Delta})$ and H5 on exposure to glutathione (reduced form) at $37^{\circ} \mathrm{C}$ in $\mathrm{pH} 7.4$ buffer.

chemical cross-linking and polymer entanglement. The decrease in mesh size by enhanced chemical crosslinking and physical entanglement of polymers thus results in a slow release of BSA from hydrogel $\mathrm{H} 8$. At $\mathrm{pH}$ 2.0 , the release of $\mathrm{BSA}$ from hydrogel $\mathrm{H} 8$ was further retarded owing to reduced swelling and probably expanded hydrodynamic volume of the protein (conformational change) at $\mathrm{pH} 2.0$.

In this study, reduction-induced degradable and $\mathrm{pH} /$ temperature-responsive hydrogels were prepared from cross-linking reactions of polymeric precursors with the bifunctional disulfide agent, cystamine, for potential applications as a colonic drug delivery matrix system. Polymeric precursors comprising NIPAAm, BA, AAc, and MAGlyGlyONp were synthesized. The structures of the copolymers were characterized by FT-IR whereas compositions and average molecular weights were evaluated mainly by ${ }^{1} \mathrm{H}$ NMR and GPC, respectively. Equilibrium swelling of hydrogels varied strongly with content of AAc at $\mathrm{pH}$ above 6.0 while little significant difference was observed at $\mathrm{pH} 2.0$. Hydrogels underwent volume change in response to change in temperature and reduction in swelling at elevated temperature was primarily determined by the content of NIPAAm. Swelling reversibility of hydrogels was observed after repeated changes in $\mathrm{pH}$ between 4.0 and 5.0. Cross-linking density was estimated by mechanical measurements and the results indicate that both polymer and cystamine concentrations influenced the degree of network formation. The authors demonstrated the degradability of hydrogels by 2-mercaptoethanol, L-cysteine and DTT. However, rapid deswelling occurred on exposure of gels to glutathione. Enhanced release of BSA was observed at $\mathrm{pH} 7.4$ for hydrogels containing sufficient amount of AAc whereas the diffusion of BSA was significantly retarded with increase in polymer concentration and chemical cross-linking of hydrogels.

Acknowledgment. Financial support from National

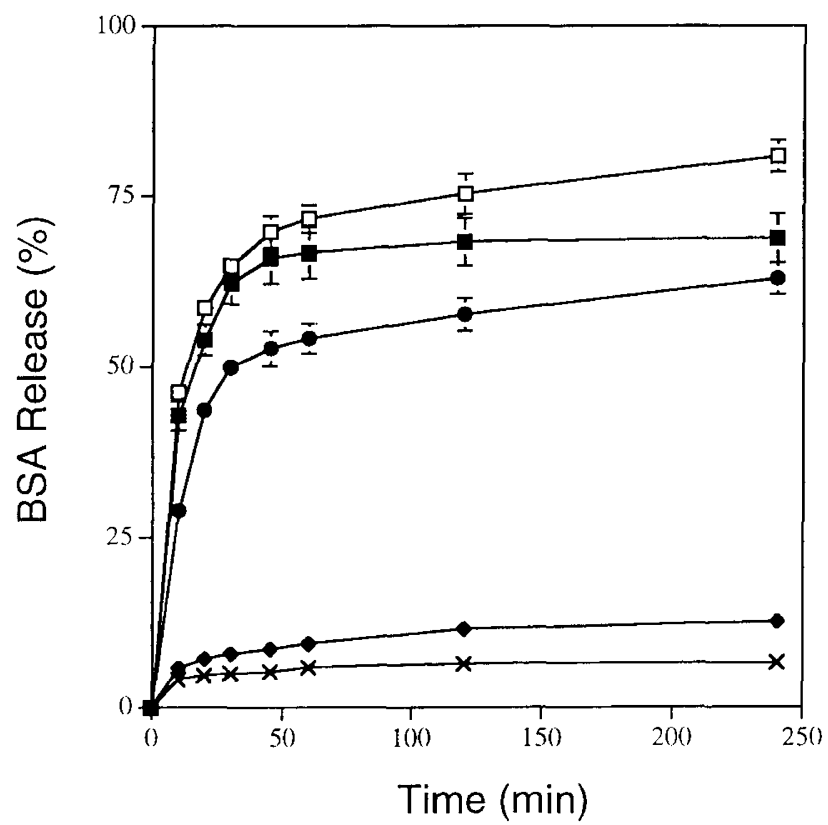

Figure 8. Cumulative release of BSA from hydrogels $\mathrm{H} 1(\square), \mathrm{H} 2$ $(\square), \mathrm{H} 3(\bigcirc)$ and $\mathrm{H} 8(\bullet)$ in $\mathrm{pH} 7.4$ buffer and $\mathrm{H} 8(\times)$ in $\mathrm{pH} 2.0$ buffer at $37^{\circ} \mathrm{C}$. Experiment performed in duplicate.

Science Council, Taiwan, Republic of China (NSC 892213-E-005-016) is gratefully acknowledged.

\section{REFERENCES}

1. D. Brown, Y. H. Bae, and S. W. Kim, Macromolecules, 27, 4952 (1994).

2. C. Wang, R. J. Stewart, and J. Kopecek, Nature, 397, 417 (1999).

3. P. W. Petka, J. L. Harden, K. P. Mcgrath, D. Wirtz, and D. A. Tirrell, Science, 281, 389 (1998).

4. T. G. Park, Biomaterials, 20, 517 (1999).

5. A. P. Sassi, A. J. Shaw, S. M. Han, H. W. Blanch, and J. W. Prausnitz, Polymer, 37, 2151 (1996).

6. M. Sen, O. Kantoglu, and O. Guven, Polymer, 40, 913 (1999).

7. H.-C. Chiu, G.-H. Hsiue, Y.-P. Lee, and L.-W. Huang, J. Biomater. Sci. Polymer Ed., 10, 591 (1999).

8. R. Yoshida, K. Uchida, Y. Kaneko, K. Sakai, A. Kikuchi, Y. Sakurai, and T. Okano, Nature, 374, 240 (1995).

9. G. Chen and A. Hoffman, Nature, 373, 49 (1995).

10. P.-Y. Yeh, P. Kopeckova, and J. Kopecek, J. Polym. Sci., Part A: Polym. Chem., 32, 1627 (1994).

11. Y. Chujo, K. Sada, A. Naka, R. Nomura, and T. Saegusa, Macromolecules, $\mathbf{2 6}, 883$ (1993).

12. Y. Chujo, K. Sada, and T. Saegusa, Macromolecules, 26, 6320 (1993).

13. Y. Chujo, K. Sada, and T. Saegusa, Macromolecules, 26, 6315 (1993).

14. W. N. E. van Dijk-Wolthuis, O. Franssen, H. Talsma, M. J van Steenbergen, J. J. Kettenes-van den Bosch, and W. E. Hennink, Macromolecules, 28, 6317 (1995).

15. L. Vervoort, G. Van den Mooter, P. Augustijns, R. Busson, S. Toppet, and R. Kinget, Pharm. Res., 14, 1730 (1997).

16. P. Rejmanova, J. Labsky, and J. Kopecek, Makromol. Chem., 178, 2169 (1979).

17. S. K. Vakkalanka, C. S. Brazel, and N. A. Peppas, J. Biomater. Sci. Polymer Ed., 8, 119 (1996).

18. H.-C. Chiu, S. Zalipsky, P. Kopeckova, and J. Kopecek, Bioconjugate Chem., 4, 290 (1993).

19. L.-C. Dong and A. S. Hoffman, J. Contr. Rel ., 15, 141 (1991).

20. E. F. Cluff, E. K. Gladding, and R. Praiser, J. Polym. Sci., 45, 341 (1960).

21. K. Ulbrich, K. Dusek, M. Ilavsky, and J. Kopecek, Eur. Polym. Sci., 14, 45 (1978). 
22. K. Dusek and W. Prins, Adv. Polym. Sci., 6, 1 (1969).

23. S. L. Snider and P. Z. Sobocinski, Analytic Biochem ., 64, 284 (1975).

24. N. A. Peppas and A. R. Khare, Adv. Drug Delivery Rev., 11, 1 (1993).

25. M. Kurisawa, M. Terano, and N. Yui, J. Biomater. Sci. Polymer Ed., 8, 691 (1997).

26. L. Hovgaard and H. Bronsted, J. Contr. Rel., 36, 159 (1995)

27. M. Heskin and J. E. Guillet, J. Macromolec. Sci. Chem., A2, 1441 (1968).

28. K. Kubota, S. Fujishige, and I. Ando, J. Phys. Chem., 94, 5154
(1990)

29. R. Yoshida, K. Sakai, T. Okano, and Y. Sakurai, J. Biomater Sci. Polymer Ed., 3, 243 (1992).

30. L. C. Dong and A. S. Hoffman, J. Contr. Rel., 4, 223 (1986).

31. N. Peppas, in "Hydrogels in Medicine and Pharmacy", Vol. 1, CRC Press, Boca Raton, FL, 1987, p 55.

32. H. Bronsted and J. Kopecek, Biomaterials, 12, 584 (1991).

33. H. Ghandehari, P. Kopeckova, and J. Kopecek, Biomaterials, 18, 861 (1997).

34. N. A. Peppas, H. J. Moynihan, and L. M. Lucht, J. Biomed. Mater. Res., 19, 397 (1985). 